\title{
Performance of the star-shaped flyer in the study of brittle materials: Three dimensional computer simulations and experimental observations
}

\author{
H. D. Espinosa, ${ }^{\text {a) }}$ G. Raiser, R. J. Clifton, and M. Ortiz \\ Division of Engineering, Brown University, Providence, Rhode Island 02912
}

(Received 27 March 1992; accepted for publication 30 June 1992)

\begin{abstract}
A three dimensional finite element computer simulation has been performed to assess the effects of release waves in normal impact soft-recovery experiments when a star-shaped flyer plate is used. Their effects on the monitored velocity-time profiles have been identified and their implications in the interpretation of wave spreading and spall signal events highlighted. The calculation shows that the star-shaped flyer plate indeed minimizes the magnitude of edge effects. The major perturbation to the one-dimensional response within the central region of the target plate results from spherical waves emanating from the corners of the star-shaped plate. Experimental evidence of the development of a damage ring located in coincidence with the eight entrant corners of the flyer plate is reported. Microscopy studies performed in the intact recovered samples revealed that this damage ring eliminates undesired boundary release waves within the central region of the specimen. Consequently, the observed damage in compression and tension within this region can be attributed primarily to the conditions arising from a state of uniaxial strain.
\end{abstract}

\section{INTRODUCTION}

The objective of a soft-recovery plate impact experiment is to be able to attribute the observable residual effects (e.g., microcrack density, dislocation configurations, phase transformed material) primarily to the stressinduced inelasticity developed during the known stress pulses of uniaxial strain.

In any plate impact experiment, due to the finite size of the plates, particles at lateral boundaries are not constrained against lateral motion. This produces waves of stress release which propagate inward and progressively eliminate the state of uniaxial strain at the center of the specimen. One of the first attempts to limit the effects of such unloading waves was the addition of a concentric spall ring around the circumference of the target plate (Smith ${ }^{1}$ and Hartman ${ }^{2}$ ). The main disadvantage of this technique was the requirement of small tolerances in the machining of the plates that made its application impractical for some brittle materials. More recently Kumar and Clifton, ${ }^{3}$ by using a star-shaped flyer (see Fig. 1 ), were able to subject LiF samples to a well defined stress history followed by a controlled release of stresses. Their main idea is to keep the central octagonal region. of the target plate relatively free from the effects of lateral unloading waves. Cylindrical, spherical, and conical waves are generated at the flyer boundary (see detailed discussion in Kumar and Clifton $^{3}$ ) directing most of the energy associated with the side rarefactions to regions at the periphery of the target plate and hence away from its center. The only cylindrical wave which passes through the central octagonal region is a shear wave diffracted from the boundary upon the arrival of a cylindrical unloading wave at $45^{\circ}$. Diffracted spherical

a) Currently at School of Aeronautics and Astronautics, Purdue University, West Lafayette, IN 47907. waves emanating from the corners of the flyer constitute the principal unloading waves that perturb the state of uniaxial strain within the central region. These features have been confirmed by Rabie et al., ${ }^{4}$ through threedimensional numerical simulations of the dynamic event, when a star-shaped target plate is used. More recently Kirkpatrick et al. ${ }^{5}$ through three-dimensional (3-D) calculations of plate-impact tensile damage experiments, have found that star-shaped target plates are preferable to circular target plates because the star geometry eliminates a late time amplification of the input pulse. Similarly, the star-shaped design has been found to be efficient for the experimental study of ceramic materials (Longy and Cagnoux ${ }^{6}$ ).

The observation of tensile cracks (Chang et $a l^{7}$ ) formed normal to the free edges of square brittle specimens when a star-shaped flyer is used, was explained by means of finite element simulations. They showed that in-plane tensile stresses are induced on the back face of the sample. This effect was attributed to size mismatch between the flyer and the sample.

The purpose of this article is to clarify the effectiveness of the soft-recovery plate impact configuration in the characterization of brittle materials. Three-dimensional finite element simulations and plate impact results obtained in the study of $\mathrm{Al}_{2} \mathrm{O}_{3}$ (Clifton et al. ${ }^{8}$ ) and AlN/AIN/Al composite (Espinosa and Clifton ${ }^{9}$ ) are used to show unambiguously the effects of unloading waves within the central region of the sample.

\section{THREE-DIMENSIONAL NUMERICAL SIMULATION}

\section{A. Numerical scheme}

Consider a body $B$ with volume $\mathrm{V}$ and surface $S$ $=S_{\sigma} \cup S_{u}$ where $S_{\sigma}$ and $S_{u}$ are the traction and kinematic 


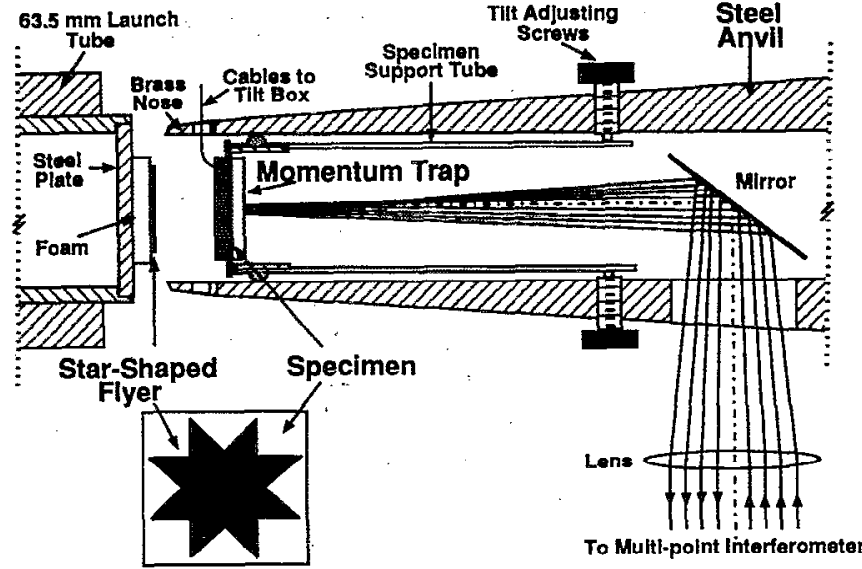

FIG. 1. Soft recovery normal impact configuration.

boundaries, respectively, and $S_{\sigma} \cap S_{u}=\varnothing$. For the small strain formulation, the strain displacement equations are

$$
\epsilon_{i j}=\left(u_{i, j}+u_{j, i}\right) / 2 \text { in } V \text {, }
$$

where $\epsilon_{i j}$ is the small strain tensor. The equations of momentum balance and boundary conditions are

$$
\begin{aligned}
& \sigma_{i j j}+\rho b_{i}=\rho \ddot{u}_{i} \text { in } V, \\
& \sigma_{i j} n_{j}=\bar{t}_{i} \text { on } S_{\sigma}, \\
& u_{l}=\bar{u}_{i} \text { on } S_{u},
\end{aligned}
$$

where $\sigma_{i j}$ is the Cauchy stress, $b_{i}$ is the body force per unit mass, $\rho$ is the density of the medium, $t_{t}$ are the surface tractions, and $u_{i}$ are the displacements. A superposed dot denotes differentiation with respect to time and a superposed bar denotes prescribed values. The formulation is complete when initial conditions are specified in the body, i.e.,

$$
u_{i}(x, 0)=u_{i}^{0} \quad \dot{u}_{i}(x, 0)=v_{i}^{0} \quad \text { in } \dot{V} .
$$

The material behavior is defined through constitutive equations which relate the motion to stress and/or stress rates. For the case of interest here, elastic response is assumed. The linear elastic constitutive equation is given by

$$
\begin{aligned}
& \sigma_{i j}=D_{i j k \epsilon} \epsilon_{k l} \\
& D_{i j k l}=\frac{\partial^{2} W}{\partial \epsilon_{i j} \partial \epsilon_{k l}},
\end{aligned}
$$

where $D$, the tensor of elastic moduli, satisfies $D_{i j k l}=D_{j i k l}$ $=D_{i j k}=D_{k i j}$

The equation of motion ( $2 a$ ) can be expressed in weak form by the principle of virtual work:

$$
\int_{V}\left[\sigma_{i j} \delta \epsilon_{i j}+\rho\left(\ddot{u}_{i}-b_{i}\right) \delta u_{i}\right] d V=\int_{S_{\bar{\sigma}}} t_{i} \delta u_{i} d S,
$$

where the variational field $\delta u_{i}$ is zero on $S_{u}$.

Due to limitations in computational resources, difficulties in the spatial resolution of the finite element discretization arise when 3-D wave propagation problems are solved. In order to reduce the domain that needs to be discretized while conserving nearly all of the physics of the real problem, energy absorbing boundaries have been developed and successfully implemented for the analysis of dynamic problems involving infinite continuous systems (Lysmer and Kuhlemeyer ${ }^{10}$ ). The efficiency of viscous boundaries has been studied by Cohen and Jennings. ${ }^{11}$ They observed that one major weakness of the silent boundary method is in the numerical noise generated upon arrival of a main stress pulse. They used numerical damping as a technique for filtering high-frequency noise. This viscous boundary approach has been incorporated in the dynamic finite element formulation with the aim of simulating the dynamic response of the momentum trap placed behind the specimen (see Fig. 1). In addition, a modification of the standard explicit integration of the governing equations is implemented. This modification removes the pathological behavior of the silent boundary method when high frequency response is required.

The tractions at the nonreflecting interface between the spccimen and the momentum trap have normal and tangential components which are taken to be

$$
\sigma_{n}=-\rho c_{d} v_{n}, \quad \sigma_{t_{1}}=-\rho c_{s} v_{t_{1}} \quad \sigma_{t_{2}}=-\rho c_{s} v_{t_{2}},
$$

where $c_{d}$ and $c_{s}$ are the dilatational and shear wave speeds, and $v_{n}, v_{t_{1}}$, and $v_{t_{2}}$ are the normal and tangential velocity components. These boundary conditions are exact for plane wave propagation across the boundary. For problems in which the wave amplitudes vary along the boundary, the use of Eq. (6) corresponds to the use of a local plane wave approximation of the wave propagation across the boundary.

By using the standard finite element spatial discretization (Hughes ${ }^{12}$ ) the weak form of the momentum balance reduces to a system of ordinary differential equations in time, i.e.,

$$
\mathbf{M u}=\mathbf{f}+\widetilde{\mathbf{f}}-\mathbf{F},
$$

where

$$
\begin{aligned}
& \mathbf{M}=\int_{V} \rho \mathbf{N}^{T} \mathbf{N} d V, \\
& \mathbf{f}=\int_{S_{\bar{\sigma}}} \mathbf{N}^{T} \mathbf{t} d S, \\
& \widetilde{\mathbf{f}}=\int_{S_{\sigma^{\mathrm{nI}}}} \mathbf{N}^{T} \mathrm{t}^{\mathrm{nr}} d S, \\
& \mathbf{F}=\int_{V} \mathbf{B}^{T} \boldsymbol{\sigma} d V,
\end{aligned}
$$

in which the traction boundary $S_{\sigma}$ has been separated into the standard traction boundary $S_{\bar{\sigma}}$ and the nonreflecting boundary $S_{\sigma} \mathrm{nr}, \mathrm{B}$ is the strain-displacement matrix, and $\mathbf{N}$ is the shape function matrix. The numerical algorithm given in Box 1 results when the explicit central difference scheme is used in the integration of Eq. (7). Corrected accelerations at step (iii) need to be computed. These correction terms arise from changes in the applied boundary 
Box 1: Explicit integration of the equations of motion

(i) initial conditions:

$n=0$

$\mathbf{u}^{0}=\overline{\mathbf{u}}^{0}, \quad \mathbf{v}^{0}=\overline{\mathbf{v}}^{0}$

$a^{0}=M^{-1}\left(f^{0}+\tilde{f}^{0}-F^{0}\right)$.

(ii) Compute nodal external forces on $S_{\bar{\sigma}}$ and $S_{\sigma}$ n:

$\tilde{\mathrm{f}}^{n+1} \equiv-\rho c \mathbf{v}^{n}$.

(iii) Correct accelerations due to changes in boundary

forces:

$\hat{\mathrm{a}}^{n}=\mathrm{a}^{n}+\mathrm{M}^{-1}\left(\widetilde{\mathbf{f}}^{n+1}-\widetilde{\mathbf{f}}^{n}\right)$ on $S_{\sigma}$ nr

$\hat{\mathbf{a}}^{n}=\mathbf{a}^{n}+\mathbf{M}^{-1}\left(\mathbf{f}^{n+1}-\mathbf{f}^{n}\right)$ on $\hat{S}_{\bar{\sigma}}$.

(iv) Update displacements:

$\mathrm{u}^{n+1}=\mathrm{u}^{n}+\Delta t \mathrm{v}^{n}+\frac{1}{2} \Delta t^{2} \hat{\mathrm{a}}^{n}$.

(v) Update the stress tensor $\sigma$ at each element.

(vi) Compute internal force vector:

$\mathbf{F}^{n+1}=\int_{V^{2}} \mathbf{B} \sigma^{n+1} d V$.

(vii) Solve for accelerations:

$\mathbf{a}^{n+1}=\mathbf{M}^{-1}\left(\mathbf{f}^{n+1}+\tilde{\mathbf{f}}^{n+1}-\mathbf{F}^{n+1}\right)$.

(viii) Update velocity vector:

$\mathrm{v}^{n+1}=\mathrm{v}^{n}+\frac{\Delta t}{2}\left(\hat{\mathrm{a}}^{n}+\mathrm{a}^{n+1}\right)$.

(ix) $n=n+1$, if $n<n_{\max }$ go to step (ii), else stop.

tractions, either due to changes in the applied external forces or due to changes in particle velocity at the nonreflecting boundary. If such corrections are not incorporated in the numerical implementation, spurious oscillations are introduced with magnitudes proportional to the traction change. These corrections are of special significance in the solution of shock wave problems, making this modification of the standard explicit algorithm an essential condition for the computation of accurate field variables. The conceptual modification in the calculation of the field variables at the boundary due to changes in the boundary tractions can be used in a variety of structural and micromechanical problems. Problems involving contact between viscoplastic bodies or problems where dynamic running cracks cause a sudden release of nodal forces are examples.

Inversion of the system of Eq. (7) is trivial when a lumped mass matrix is used, minimizing the computational effort per time step. The method is conditionally stable, the stability criterion is (Hughes ${ }^{12}$ )

$$
\Delta t<\frac{2}{\omega_{\max }},
$$

where $\omega_{\max }$ is the maximum frequency of the finite element structure. Reducing the time step to values below $\Delta t_{\mathrm{cr}}$ leads to problems of dispersion arising from the spatial errors introduced by the finite element discretization. For the special case of one-dimensional uniform meshes, when $\Delta t$ is chosen such that the Courant number $\left(q=\Delta t c_{d} / l\right.$, where $l$ is the element size) is equal to 1 , there is no numerical

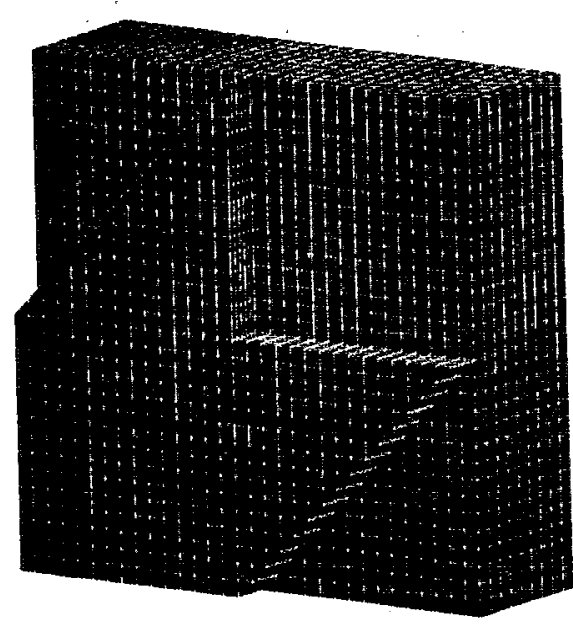

FIG. 2. Star-shaped flyer and specimen configuration used in the 3-D calculations.

dispersion. This result suggests that meshes should be made as uniform as possible.

\section{B. Numerical results}

A plot of the mesh composed of 8-node, isoparametric brick elements is given in Fig. 2. To simulate the flyer shape, the brick elements at the boundary are collapsed onto one face. A total of 22000 elements in the target plate and 4000 elements in the flyer plate are used. By symmetry only one quadrant is discretized, symmetry conditions are imposed on the left and lower faces. The target dimensions are $23 \mathrm{~mm} \times 23 \mathrm{~mm} \times 4 \mathrm{~mm}$, the fiyer plate has matching dimensions and a thickness of $1 \mathrm{~mm}$. The material properties for the $\mathrm{Al}_{2} \mathrm{O}_{3}$ specimen and the $\mathrm{Al}$ flyer are given in Table I. The interface between the flyer and target plates has been modeled such that separation of the surfaces can take place. The initial conditions are prescribed as follows: (i) nodes at the momentum trap and inside the specimen are considered to be at rest, (ii) nodes at the flyer-specimen interface are initialized with the velocity obtained from an elastic solution according to the characteristic equations, and (iii) nodes within the flyer are initialized with a velocity equal to the projectile velocity.

The particle velocity versus time profile at four different points located at the back of the specimen are shown in Figs. 3(a)-3(d). Finite risetimes at wave fronts and small oscillations are the result of geometric dispersion effects associated with the spatial discretization. Figures 3(a)3(c) show a compressive pulse with constant amplitude

TABLE I. Material properties.

\begin{tabular}{|c|c|c|c|c|c|}
\hline Material & $\begin{array}{c}\text { Density } \\
\left(\mathrm{kg} / \mathrm{cm}^{3}\right)\end{array}$ & $\begin{array}{l}\text { Longitudinal } \\
\text { wave speed } \\
(\mathrm{mm} / \mu \mathrm{s})\end{array}$ & $\begin{array}{c}\text { Transverse } \\
\text { wave speed } \\
(\mathrm{mm} / \mu \mathrm{s})\end{array}$ & $\begin{array}{c}\text { Acoustic } \\
\text { impedance } \\
(\mathrm{GPa} / \mathrm{mm} / \mu \mathrm{s})\end{array}$ & $\begin{array}{c}\text { Shear } \\
\text { impedance } \\
(\mathrm{GPa} / \mathrm{mm} / \mu \mathrm{s})\end{array}$ \\
\hline Aluminum 6061-T6 & 2700 & 6.4 & 3.05 & 17.34 & 8.66 \\
\hline $\mathrm{Al}_{2} \mathrm{O}_{3}$ & 3990 & 10.8 & 6.4 & 43.09 & 25.54 \\
\hline
\end{tabular}




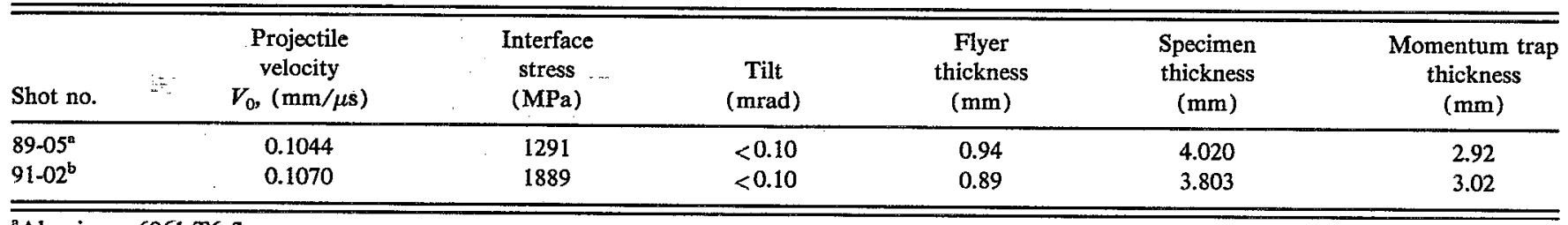

${ }^{2}$ Aluminum 6061-T6 flyer.

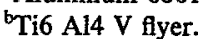

proportional to the impact velocity. Three dimensional wave contributions arrive after the end of the initial pulse, their magnitude is of the order of $8 \%$ of the initial pulse velocity. Figure 3(d), which corresponds to a position outside the central octagonal region, shows the effects of the unloading wave almost immediately after the arrival of the front. Evidence of such response is observed as a departure from the 1-D elastic prediction.

Stress-time profiles at the center of the target plate, at depths $0, h / 2$, and $h$ (where $h$ is the thickness of the spec- imen), are given in Fig. 4. After an initial compressive state of stress, tensile stresses with maximum amplitude on the order of $15 \%$ of the longitudinal compressive stress in the incident plane wave, are developed at the rear surface of the specimen. The first peak (at $t \simeq 1.1 \mu \mathrm{s}$ ) corresponds to the simultaneous arrival of the eight diffracted spherical waves emanating from the re-entrant corners. The second peak (at $t \simeq 1.7 \mu \mathrm{s}$ ), which represents a maximum, is produced by the superposition of unloading waves generated at the eight corners located at the periphery of the star-
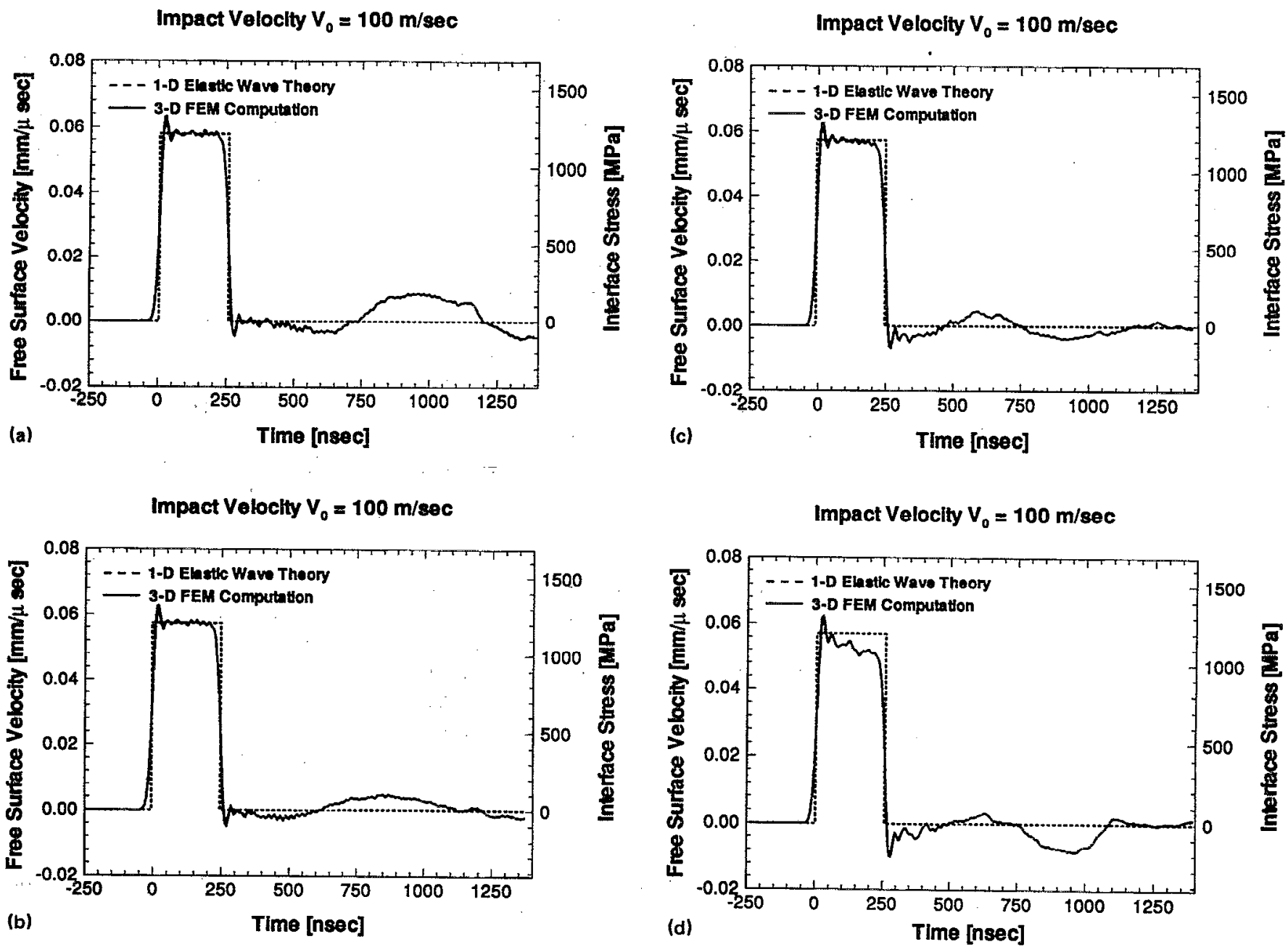

FIG. 3. (a) Velocity-time profile at the center of the specimen. (b) Velocity-time profile at a point $1.95 \mathrm{~mm}$ to the right of the specimen center. (c) Velocity-time profile at a point $4.5 \mathrm{~mm}$ to the right of the specimen center. (d) Velocity-time profile at a point $5.85 \mathrm{~mm}$ to the right of the specimen center. 


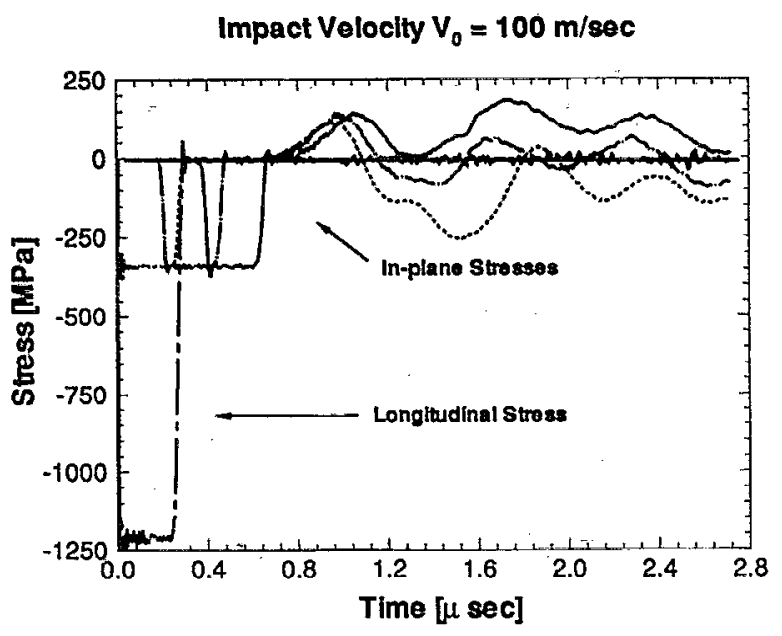

FIG. 4. Normal and in-plane stress-time profiles at the center of the target plate. In-plane stress histories at depths $0, \frac{h}{2}$, and $h$.

shaped plate. The duration of these tensile waves is determined by the duration of the initial compressive pulse. These tensile stresses are the result of unloading waves independent of any size mismatch between the flyer and the target plates.

The computer simulation reported here corresponds to the worst scenario where unloading waves from the eight corners of the star-shaped flyer are produced simultaneously, i.e., no tilt between the flyer and the target. Moreover, as discussed by Kumar and Clifton, ${ }^{3}$ the maximum amplitude of the release waves decreases with decreasing ratio $l / r$, where $l$ is the flyer thickness and $r$ is the distance from the reentrant corner. This fact should be used in a correct design of the recovery experiment. Another reason for the need of a thin flyer arises from the generation of residual transverse strains upon shock release in materials that undergo plastic deformation or phase transition under conditions of uniaxial strain (Stevens and Jones ${ }^{13}$ ).

\section{EXPERIMENTAL OBSERVATIONS}

Velocity-time profiles obtained using the soft-recovery configuration in $\mathrm{Al}_{2} \mathrm{O}_{3}$ (Clifton et al. ${ }^{8}$ ) and $\mathrm{AlN} / \mathrm{AlN} / \mathrm{Al}$ (Espinosa and $\mathrm{Clifton}^{9}$ ) for the highest imposed impact velocities, are given in Figs. 5 and 6, respectively. A summary of the experiments is given in Table II. The wave structure observed between the first and the second compressive pulses, in the $\mathrm{Al}_{2} \mathrm{O}_{3}$ experimental record (Fig. 5), is caused in part by wave spreading during damage in compression and in part from release waves emanating from the corners of the fiyer plate. In the AlN/AlN/Al experimental record (Fig. 6), the additional feature of reflected waves originating at the tensile damage (spall) region is observed immediately after the initial compressive pulse. These observations together with the results of the 3-D computer simulation lead to the conclusion that proper interpretation and quantification of the velocity-time histories require the consideration of the release waves con-

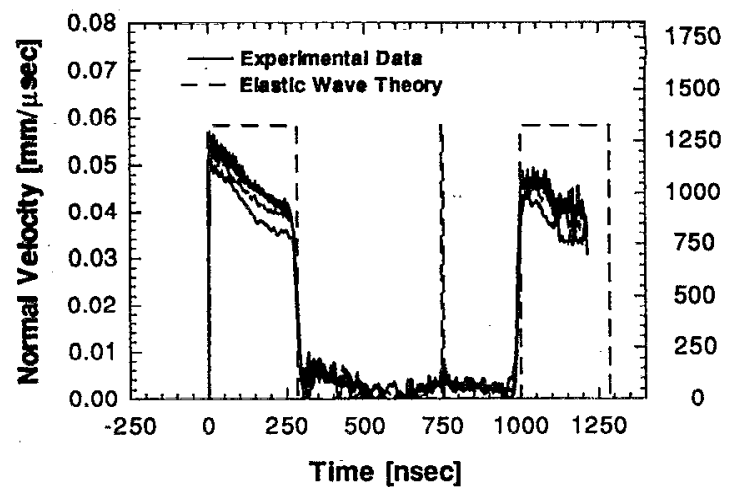

要

FIG. 5. Monitor velocity-time profiles at four points within the central region of the $\mathrm{Al}_{2} \mathrm{O}_{3}$ sample (shot 89-05).

tribution to the normal velocity amplitude. Two pictures of the recovered $\mathrm{Al}_{2} \mathrm{O}_{3}$ sample are shown in Fig. 7. At the impact surface of the specimen, Fig. 7(a), high density of microcracks and a few macrocracks can be observed outside the central region. This damage pattern is consistent with the tensile stresses induced by the unloading cylindri$\mathrm{cal}$ and spherical waves. At the rear face of the specimen, Fig. 7(b) a damage ring located in coincidence with the eight entrant corners of the flyer plate is also observed. The divergent character of the unloading waves from the corners makes these locations the most favorable regions for stress-induced microcracking; consequently, the level of tensile stresses that propagates into the central octagonal region is attenuated to a value below a fracture stress threshold. The net effect is the generation of a protective ring that eliminates undesired effects during the generation of the main compressive pulse.

Recovered AlN/AIN/Al samples, with a fracture toughness approximately twice the $K_{I C}$ of $\mathrm{Al}_{2} \mathrm{O}_{3}$, exhibit the same general features. A SEM micrograph of the spall region within the sample is shown in Fig. 8. The only main cracks developed inside the central region of the sample are perpendicular to the direction of impact, consistent with the expected state of uniaxial strain.

The observation of cross-shaped cracks by Chang et al. ${ }^{7}$ in the study of Mg-PSZ seems to be the result of the

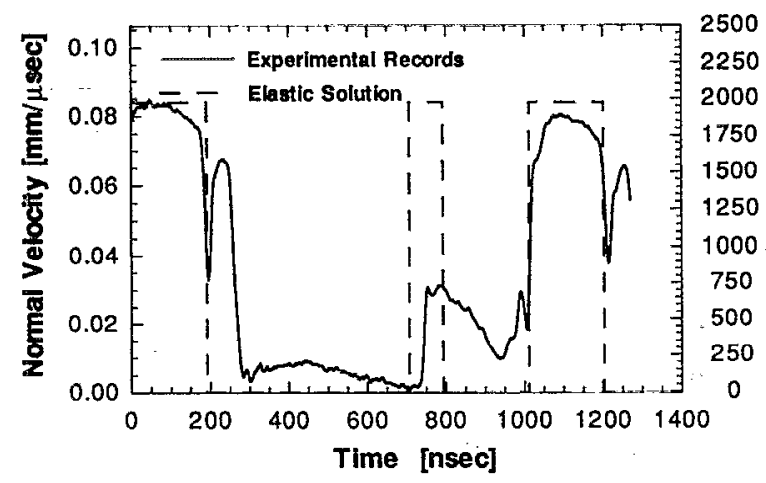

疍

FIG. 6. Monitor velocity-time profile in AlN/AIN/Al (shot 91-02). 

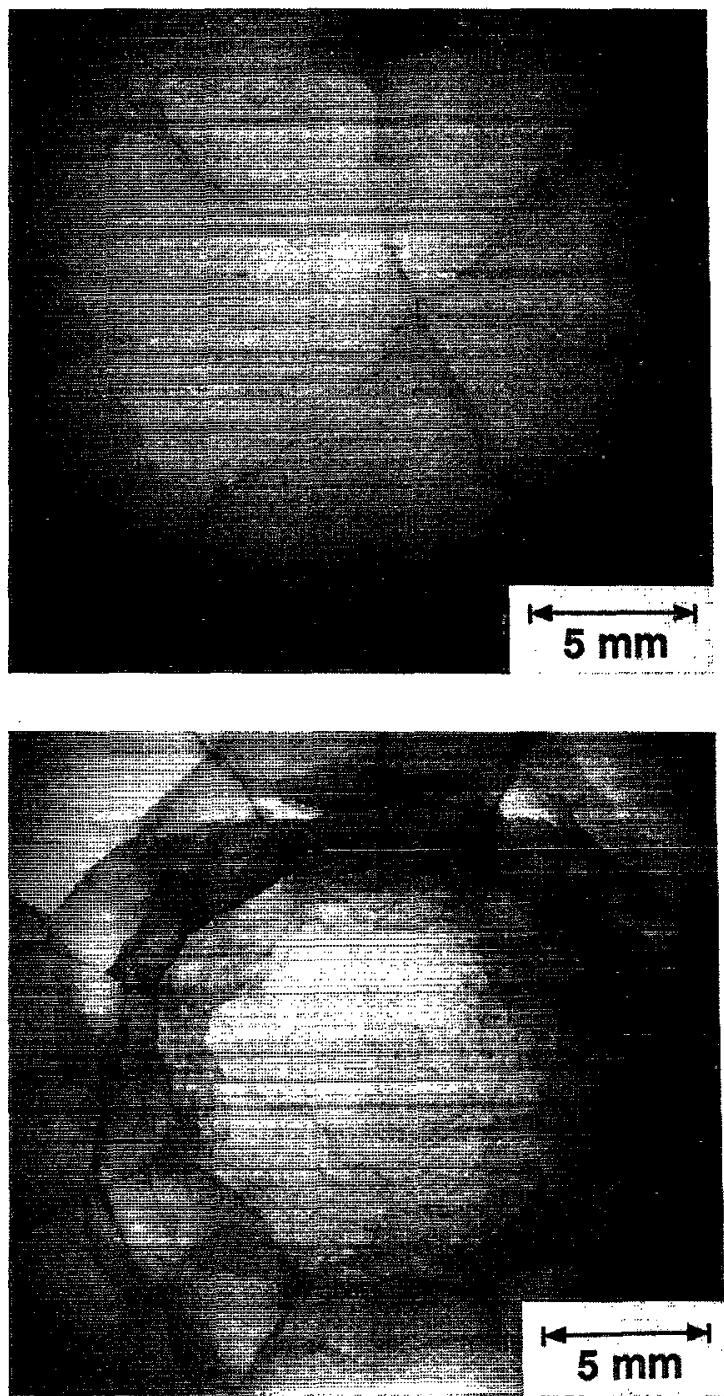

FIG. 7. (a) Microcrack pattern at the impact face of the $\mathrm{Al}_{2} \mathrm{O}_{3}$ sample. (b) Microcrack pattern at the back face of the $\mathrm{Al}_{2} \mathrm{O}_{3}$ sample.

$1 \mu$ s applied pulse (magnifying the spherical wave effects) and the pre-existence of $200-300 \mu \mathrm{m}$ surface flaws. These flaws appear to have provided the microcrack nuclei for propagation of main localized cracks within the central region of the specimen. Another possible source of tensile stresses, not explored by the authors, is the additional dissipation of elastic energy trapped in the central region of the sample upon unloading of the longitudinal compressive pulse. This energy arises as a consequence of inelastic deformations that result from the stress-induced phase transformation in the Mg-PSZ.

\section{CONCLUSIONS}

Three-dimensional effects due to release waves in the soft-recovery configuration proposed by Kumar and Clifton, ${ }^{3}$ have been quantified through elastic finite element simulations. The effects of release waves on the monitored velocity-time profiles have been assessed and their implica-

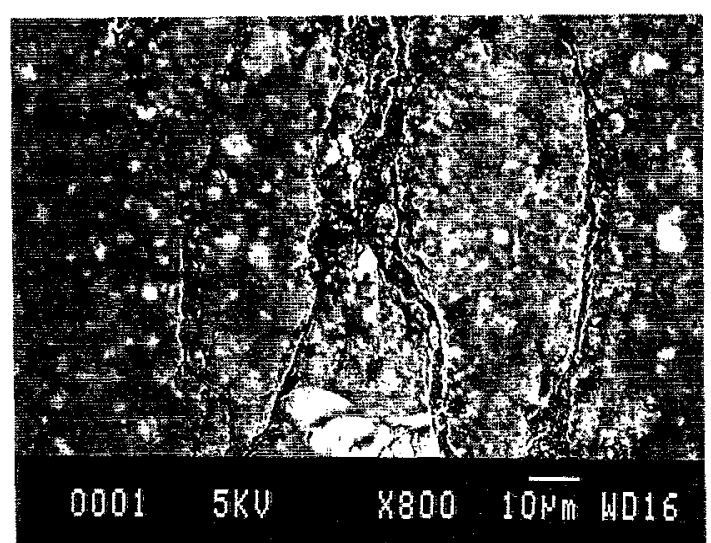

FIG. 8. SEM micrograph showing the spall region developed within the central region of the AIN/AIN/Al specimen. Note that the main cracks are perpendicular to the direction of wave propagation.

tions for the interpretation of wave spreading and spall signals have been noted. Transverse tensile stresses calculated for the back of the sample have been identified as being due primarily to diffracted spherical waves emanating from the corners of the flyer plate. The overall efficiency of the star-shaped flyer design in minimizing boundary release waves, up to maximum longitudinal stresses of $2 \mathrm{GPa}$, has been shown through experimental velocity-time profiles and microscopy studies for an $\mathrm{Al}_{2} \mathrm{O}_{3}$ ceramic and an AlN/AlN/Al composite.

\section{ACKNOWLEDGMENTS}

This research was supported by the National Science Foundation through its support of the MRG on Micromechanics of Failure-Resistant Materials. We acknowledge the support of Lanxide Armor Products, Inc. in providing the AlN/AlN/Al composite material samples.

'J. H. Smith, Symposium on Dynamic Behavior of Materials, ASTM Special Technical Publication 336 (American Society for Testing and Materials, Philadelphia, PA, 1963).

${ }^{2}$ W. F. Hartman, J. Appl. Phys. 35, 2090 (1964).

${ }^{3}$ P. Kumar and R. J. Clifton, J. Appl. Phys. 48, 4850 (1977).

${ }^{4}$ R. L. Rabie, J. E. Vorthman, and J. K. Dienes, Shock Waves in Condensed Matter, edited by J. R. Asay, R. A. Graham, and G. K. Straub (Elsevier Science, 1983), Vol. 5, p. 199.

${ }^{5}$ S. W. Kirkpatrick, D. R. Curran, D. C. Erlich, and R. W. Klopp, Proceedings of 1991 APS Topical Conference on Shock Compression of Condensed Matter, edited by S. C. Schmidt, J. N. Johnson, and L. W. Davison (Elsevier Science, 1992).

${ }^{6}$ F. Longy and J. Cagnoux, J. Am. Ceram. Soc. 72, 971 (1989).

${ }^{7}$ S. N. Chang, D. T. Chung, G. Ravichandran, and S. Nemat-Nasser, Proceedings of 1989 APS Conference on Shock Compression of Condensed Matter, edited by S. C. Schmidt, J. N. Johnson, and L. W. Davison (Elsevier Science, 1990), p. 389

${ }^{8}$ R. J. Clifton, G. Raiser, M. Ortiz, and H. D. Espinosa, Proceedings of 1989 APS Conference on Shock Compression of Condensed Matter, edited by S. C. Schmidt, J. N. Johnson, and L. W. Davison (Elsevier, Science, 1990), p. 437.

${ }^{9}$ H. D. Espinosa and R. J. Clifton, ASME Winter Annual Mceting, 
Symp. on Experiments in Micromechanics of Fracture-Resistant Materials, edited by K. S. Kim (ASME, 1991), p. 37

${ }^{10} \mathrm{~J}$. Lysmer and R. L. Kuhlemeyer, J. Eng. Mech. Div. ASCE 95 (EM4) 859 (1969).

${ }^{11}$ M. Cohen and P. C. Jennings, Computational Methods for Transient
Analysis, edited by T. Belytschko and T. J. R. Hughes (Elsevier Science, 1983), p. 301.

${ }^{12}$ T. J. R. Hughes, The Finite Element Method (Prentice Hall, Englewood Cliffs, N. J., 1987).

${ }^{13}$ A. L. Stevens and O. E. Jones, J. Appl. Mech. 39, 45 (1972). 